\title{
Development and use of three-dimensional image analysis algorithms to evaluate puffing of banana slices undergone combined hot air and microwave drying
}

\section{Satienkijumpai, A. ${ }^{{ }^{*}}$; Jinorose, M. $^{\mathrm{a}^{*}}$; Devahastin, S. ${ }^{\mathrm{b}}$}

a Department of Food Engineering. Faculty of Engineering. King Mongkut's Institute of Technology Ladkrabang, 1 Soi Chalongkrung 1, Ladkrabang, Bangkok, 10520, Thailand.

b Department of Food Engineering. King Mongkut's University of Technology Thonburi, 126 Pracha u-tid Road, Tungkru, Bangkok, 10140, Thailand.

*E-mail of the corresponding author: maturada.ji@kmitl.ac.th

\begin{abstract}
Puffing is an attractive alternative for the production of healthy crisp snacks without frying. Although image analysis has been used in some prior studies to evaluate puffing, such an evaluation was made only in one or two dimensions, which is inadequate when a sample deforms in three dimensions. In this study, use of combined hot-air and microwave drying to dry and puff banana slices was first evaluated. Algorithms were then developed to characterize the changes in the appearance of puffed banana slices. Various image-based parameters, both in two and three dimensions were assessed and used to monitor the puffing.
\end{abstract}

Keywords: Deformation; Puffing; Imge analysis; Physical properties; Surface texture 


\section{Introduction}

Snacks, especially those made from fruits and vegetables, have recently received increasing attention and are widely consumed by health-conscious consumers. Such an increased consumption is due to the fact that modern consumers are paying more attention to their health and trying to switch from traditional to non-sugary and low-fat or even fat-free snacks. Low-fat or fat-free snacks, which are produced by hot air drying, however, suffer important drawbacks; these snacks generally exhibit inferior texture to those obtained via the process of frying. An alternative drying technology is clearly needed to alleviate the drawbacks. Combined hot-air and microwave drying has indeed been suggested and applied to produce dried fruit snacks with more desirable texture.[1] The superior texture is due to puffing, which occurs due to rapid expansion of the fruit microstructure as a result of rapid internal evaporation of water into vapor that cannot escape from such a microstructure at an adequate rate.

Puffing is generally evaluated and reported in terms of volumetric deformation, which is calculated as the ratio of the volume of a sample after drying to that before drying.[2] However, volumetric deformation cannot be used to describe non-uniform or irregular puffing, which normally takes place and can significantly affect the appearance and hence the consumer's acceptance of a final product.[3] It has in fact been reported that two pieces of a material may exhibit similar volumetric shrinkage (or deformation) despite the fact that they had gone through different methods of drying and clearly possess different forms (shape and size) of deformation.[4]

Although image analysis has been used in some earlier studies to evaluate deformation during drying, attempts were usually made only to evaluate deformation in one or two dimensions, which is not adequate when a sample deforms non-uniformly[3] or exhibits irregularly rugged surface in three dimensions such as in the case of puffing. This is simply because one and two dimensional imagings are much easier than three-dimensional imaging, eventhough they cannot accurately well represent deformation.[3] In addition, despite some recent attempts to describe deformation using indicators derived from imagebased information, most studies only focused on uniform deformation of simple shapes, e.g., spherical, cylindrical and cubical shape.[3] The ability to precisely identify the shape and its changes of an irregularly shaped materials remains a challenge.

In this study, the use of combined hot-air and microwave drying to dry and puff banana slices was first evaluated. Algorithms and software were developed to characterize the changes in the appearance of puffed banana slices. Various image-based parameters, both in two dimensions (i.e., projected area, major and minor axes, equivalent diameter, perimeter, fractal dimension, extents, form factor and aspect ratio) and three dimensions 
(i.e., image-based volume, surface area, sphericities, Wadell's roundness, radius ratio and Hoffmann shape entropy), were assessed and used to monitor the puffing.

\section{Materials and Methods}

\subsection{Banana slices preparation}

Banana of the Namwa variety (Musa sapientum L. (ABB group)) was used in this study. Banana was purchased from a local supermarket and kept at room temperature $\left(28^{\circ} \pm 3^{\circ} \mathrm{C}\right)$ until its total soluble solids (TSS) reached 28.5 $\pm 0.5^{\circ}$ Brix. Banana was peeled and sliced to the dimensions of $5 \pm 0.5 \mathrm{~mm}$ in thickness and $30.0 \pm 2.0 \mathrm{~mm}$ in diameter; the dimensions were measured by a Vernier caliper (Winston, Japan).

\subsection{Drying and puffing experiments}

Drying experiments were conducted in a convective hot-air dryer (Memmert, UF30, Germany) at $70^{\circ} \pm 5^{\circ} \mathrm{C}$ until the banana moisture content reached either $10,20,30$ or $40 \%$ dry basis (d.b.). Puffing was then conducted in a domestic microwave oven (Samsung, MS23K3513AW, Malaysia) at an input powder of $800 \mathrm{~W}$ for either 0, 20, 40 or $60 \mathrm{~s}$.

\subsection{Image acquisition}

Two-dimensional images of banana slices were first taken via the use of a scanner (Epson, V30, Indonesia) at 300 dpi with black background to reduce the shadow. Threedimensional images were produced from the two-dimensional images as per the procedures developed by Jinorose et al.[3] Two-dimensional images were preprocessed by MeshLab (ISTO-CNR, Visual computing Laboratory) to reduce unwanted objects and then reconstructed using Autodesk Recap 360 software (Autodesk Inc., San Rafael, CA) into three-dimensional images.

\subsection{Image analysis}

Each 2D image was preprocessed and analyzed using MATLAB $^{\circledR}$ (version R2015b, MathWorks Inc., MA). Image segmentation was conducted by converting RGB image into binary image using Otsu's thresholding method. Edge detection and holes filling were performed to segment the area of interest (AOI). All the basic image parameters including projected area, major axis length, minor axis length, equivalent diameter, perimeter and extent were calculated using the functions of MATLAB ${ }^{\circledR}$ image processing tools box. Other parameters were also calculated as per the following equations.[5]

$$
\begin{aligned}
& \text { Extent } 2=\frac{A}{D_{\mathrm{fmax}} D_{\mathrm{fmin}}} \\
& \text { Form factor }=\frac{4 \pi A}{P^{2}}
\end{aligned}
$$


Development and use of three-dimensional image analysis algorithms to evaluate puffing of banana slices undergone combined hot air and microwave drying

$$
\text { Aspect ratio }=\frac{D_{\mathrm{fmax}}}{D_{\mathrm{fmin}}}
$$

In the case of $3 \mathrm{D}$ analysis, after a 3D image was reconstructed, the image was converted into an STL file and imported into COMSOL Multiphysics ${ }^{\circledR}$ version 3.5 (COMSOL, Inc., Sweden) to calculate the image-based volume, surface area, sphericity, Wadell's sphericity, Wadell's roundness, radius ratio and Hoffmann shape entropy.[5,6] 'fine mesh' setting was adopted when assigning meshes to the object prior to the calculation.

$$
\begin{aligned}
& \text { Sphericity }=\frac{(36 \pi)^{\frac{1}{6} V^{\frac{1}{3}}}}{S A^{\frac{1}{2}}} \\
& \text { Wadell's sphericity }=\frac{S A_{\mathrm{V}}}{S A} \\
& \text { Wadell's roundness }=\frac{4 A}{\pi D_{\mathrm{fmax}}{ }^{2}} \\
& \text { Radius Ratio }=\frac{R_{\min }}{R_{\max }} \\
& \text { Hoffmann shape entropy }=\frac{1}{\ln \left(\frac{1}{3}\right)} \sum_{i=1}^{3} p_{i} \ln p_{i}
\end{aligned}
$$

\subsection{Moisture content determination}

The moisture content of a sample was determined as per AOAC method 984.25 (2000).

\subsection{Volume determination}

The volume of a sample was determined as per the methods of Yan et al.[7] The sample was suspended in $125 \mathrm{~mL}$ of $95 \%$-heptane, which was filled in a $250-\mathrm{mL}$ beaker placed on a 3-digit balance (Want, WT3203N, China).

All experiments were performed in triplicate and the results, where appropriate, are reported as mean values and standard deviations.

\section{Results and discussion}

\subsection{Drying of banana slices}

Preliminary experiments revealed that banana slices suffered phase transition and stuck to the tray when drying was conducted at $90^{\circ} \mathrm{C}$. Drying was therefore conducted at 50 and $70^{\circ} \mathrm{C}$; the degrees of puffing (or deformation) and visual appearance were noted to be almost the same at the same moisture content either when drying was conducted at 50 or 
$70^{\circ} \mathrm{C}$. As a result, drying at $70^{\circ} \mathrm{C}$ was finally selected in the interest of time and energy conservation.

\subsection{Puffing of banana slices}

After drying to the predetermined moisture content, puffing was conducted. Selected images of the samples at different puffing time are shown in Fig. 1. Deformation occurred rather uniformly until about $40 \mathrm{~s}$, after which the sample started to deformed nonuniformly. This visually observed critical point corresponded to the moisture ratio of around 0.4-0.5 (Fig. 2a).

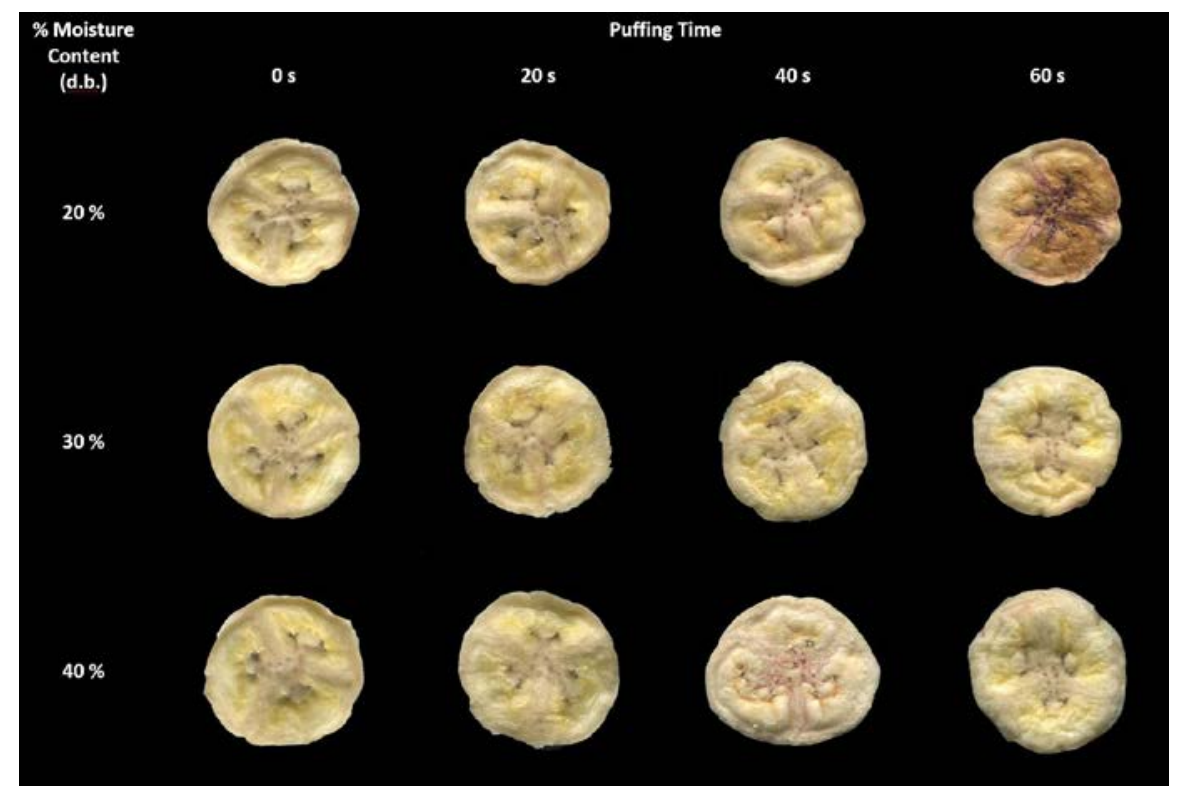

Fig. 12 D images of banana slices during puffing.

The evolution of the degree of puffing (volumetric deformation) as a function of the moisture ratio with moisture content prior to puffing as a parameter is shown in Fig. $2 \mathrm{~b}$. Degree of puffing increased linearly even when the moisture ratio reached around 0.2 (or the puffing time of around $60 \mathrm{~s}$ as seen in Fig. 2a). It is important to note that the degree of puffing, which was obtained from liquid displacement measurement, could not identify the start of the non-uniform deformation period, which took place at around $40 \mathrm{~s}$ as mentioned earlier. 


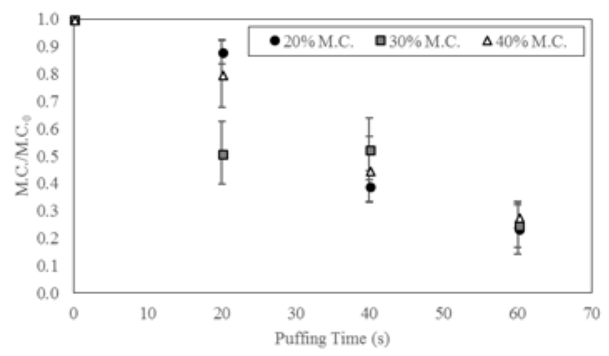

(a)

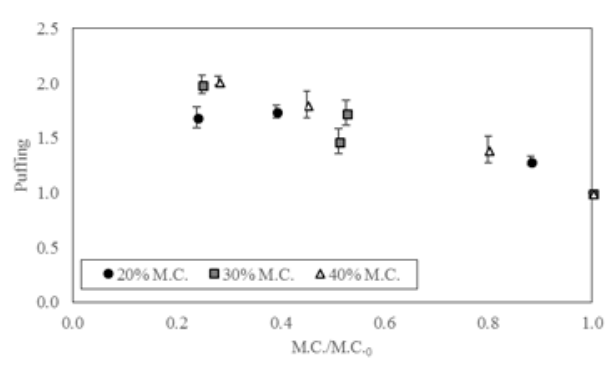

(b)

Fig. 2 (a) Moisture ratio as a function of puffing time of banana slices.

(b) Degree of puffing of banana slices as a function of moisture ratio.

\subsection{Image-based information}

Fig. 3a shows the evolutions of the 2D image-based parameters as a function of the moisture ratio of banana slices undergoing puffing. When considering which parameters could be used to describe the non-uniform deformation (or, in other words, change of shape), only the aspect ratio and extent 2 could be used. It is seen that while other parameters stayed unchanged, these two parameters started to vary significantly when the moisture ratio was around 0.5 , beyond which their values started to increase. This corresponded to the critical point where non-uniform deformation was observed to start (see Fig. 1). Nevertheless, these two parameters could only identify the onset of the nonuniform deformation but not the deformation itself. These 2D parameters could also not be used to monitor the volume change of the samples, as expected. Since puffing naturally involves the change of volume, inability to monitor such a change is clearly inadequate.

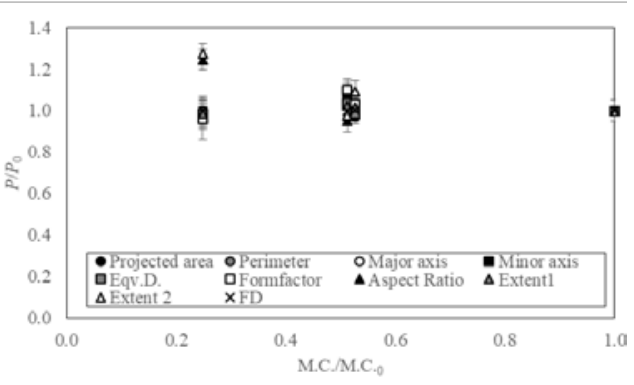

(a) 2D image-based parameters

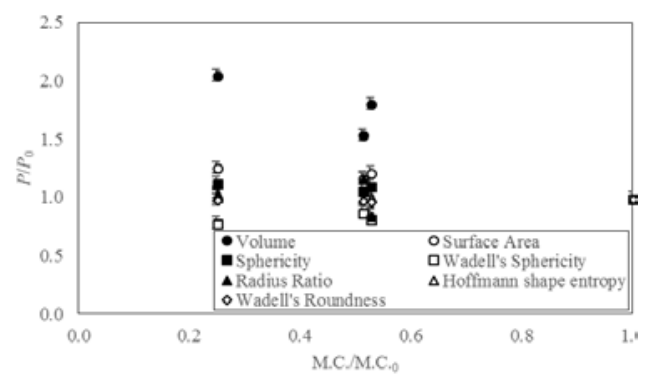

(b) 3D image-based parameters

Fig. 3 (a) Evolutions of image-based parameters as a function of moisture ratio of banana slices undergoing puffing. $P$ = parameter of interest; 0 = initial value. 
Interestingly, form factor and fractal dimension, which are widely used to indicate circularity and shape changes, remained unchanged over the whole puffing period. This incorrectly implies that banana slices retained their shape throughout the process, which contradicted to the visual observation results. This is most probably because 2D top-view images are not adequate for the evaluation of a sample with rugged surface (as in our case of puffed banana slices). For this reason, choosing appropriate parameters is very important if non-uniform deformation is to be monitored; widely used parameters cannot always be used for such a purpose.

Fig. $3 \mathrm{~b}$ shows the evolutions of the $3 \mathrm{D}$ image-based parameters as a function of the moisture ratio of banana slices undergoing puffing. The volume and surface area values were those of the reconstructed 3D images; the values increased with decreasing moisture ratio. Only the Wadell's sphericity exhibited a similar trend to the aspect ratio and extent 2 (started to decrease after the moisture ratio was lower than 0.5 ) and might be able to be used to monitor the non-uniform deformation.

Based on the aforementioned observations, volume and surface area along with the Wadell's sphericity should be used to monitor the puffing of banana slices. Fig. 4 indeed illustrates that the volume as obtained from image analysis agreed well with the values obtained from the liquid displacement experiments.

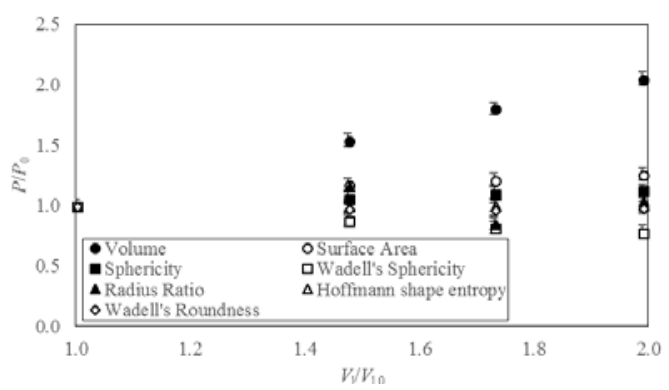

Fig. 4 Evolutions of 3D image-based parameters as a function of volume ratio of banana slices undergoing puffing. $P=$ Parameter of interest; $0=$ initial value.

\section{Conclusions}

Image analysis algorithms were developed to characterize puffing, which is a non-uniform deformation phenomenon, of banana slices in three dimensions. Various image-based parameters, both in 2D and 3D, were calculated and tested for their suitability to monitor such a deformation. As for the 2D-based parameters, aspect ratio and extent 2 could be used to indicate the start of the non-uniform deformation period; Wadell's sphericity represented the 3D-based parameters that could be used to perform the similar task. These parameters, however, could not be used to monitor the volume change of the samples. 3D-based volume 
and surface area along with the Wadell's sphericity should instead be used to monitor the puffing of banana slices.

\section{Acknowledgements}

The authors express their sincere appreciation to the Thailand Research Fund (TRF) for supporting the study financially through its New Researcher Grant awarded to Author Jinorose (Grant No. TRG 5880109) and Senior Research Scholar Grant awarded to Author Devahastin (Grant No. RTA 5880009).

\section{Nomenclature}

\begin{tabular}{|c|c|c|}
\hline$A$ & Projected area & $\mathrm{cm}^{2}$ \\
\hline$D_{\mathrm{fmax}}, D_{\mathrm{fmin}}$ & Maximum and minimum ferret diameter & $\mathrm{m}$ \\
\hline Major, Minor & Major and minor axis length & $\mathrm{cm}$ \\
\hline$P$ & Perimeter & $\mathrm{cm}$ \\
\hline$p_{\mathrm{i}}$ & $\begin{array}{l}\text { Ratio between length of each dimension to sum } \\
\text { of length of all dimension in } 3 \mathrm{D} \text { image }\end{array}$ & \\
\hline$R_{\max }$ & Radius of the smallest circumscribe in 3D image & voxel \\
\hline$R_{\min }$ & Radius of the biggest inscribe sphere in 3D image & \\
\hline$S A$ & Surface area of sample from 3D image & $\mathrm{cm}^{2}$ \\
\hline$S A_{\mathrm{v}}$ & $\begin{array}{l}\text { Surface area of sphere that have same volume } \\
\text { from 3D image }\end{array}$ & $\mathrm{cm}^{2}$ \\
\hline$V$ & Volume of sample from 3D image & $\mathrm{cm}^{3}$ \\
\hline
\end{tabular}

\section{References}

[1] Paengkanya, S.; Soponronnarit, S.; Nathakaranakule, A. Application of microwaves for drying of durian chips. Food and Bioproducts Processing 2015, 96, 1-11.

[2] Tabtiang, S.; Prachayawarakon S.; Soponronnarit, S. Effects of osmotic treatment and superheated steam puffing temperature on drying characteristics and texture properties of banana slices. Drying Technology 2012, 30(1), 20-28.

[3] Jinorose, M.; Stienkijumpai, A.; Devahastin, S. Use of digital image analysis as a monitoring tool for non-uniform deformation of shrinkable materials during drying. Journal of Chemical Engineering of Japan 2017, 50(1), 785-791.

[4] Devahastin, S.; Niamnuy, C. Modelling quality changes of fruits and vegetables during drying: a review. International Journal of Food Science and Technology 2010, 45, 1755-1767.

[5] Neal, F.B.; Russ, C.J. Measuring Shapes; CRC Press: Boca Raton, 2012; 231-290.

[6] Bullard, J.W.; Garboczi, E.J. Defining shape measures for 3D star-shaped particles: sphericity, roundness, and dimensions. Powder Technology 2013, 249, 241-252.

[7] Yan, Z.; Sousa-Gallagher, M.J.; Oliveira, F.A.R. Shrinkage and porosity of banana, pineapple and mango slices during. Journal of Food Engineering 2008, 84(3), 430-440. 